\title{
MATLAB Implementation of an Adaptive Neuro-Fuzzy Modeling Approach applied on Nonlinear Dynamic Systems - a Case Study
}

\author{
Roxana-Elena Tudoroiu \\ University of Petrosani \\ 20 Universității Street, \\ 332006, Petroșani, \\ Romania \\ tudelena@mail.com
}

\author{
Mohammed Zaheeruddin \\ Concordia University 1455 \\ De Maisonneuve Blvd West, \\ Montreal, QC, H3G 1M8, \\ Canada \\ zaheer@encs.concordia.ca
}

\author{
Dumitru Dan Burdescu \\ University of Craiova, 107 \\ Decebal Bvd., 200440, Craiova, \\ Romania \\ dburdescu@yahoo.com
}

\author{
Nicolae Tudoroiu \\ John Abbott College 21275 \\ Lake Shore Road, Sainte-Anne- \\ de-Bellevue, QC, H9X 3L9, \\ Canada \\ ntudoroiu@gmail.com
}

\begin{abstract}
In this paper one of the most accurate adaptive neuro-fuzzy modelling approach is investigated. It is suitable for modelling the nonlinear dynamics of any process or control systems. Basically, this new modelling approach is an improvement of a linear ARX polynomials models based on the least square errors estimation method that is preferred for its simplicity and faster implementation, since it uses typical functions from MATLAB system identification toolbox. For simulation purpose, to prove its effectiveness in terms of modeling accuracy, an appropriate case study of a centrifugal chiller is considered. The reason for this selection is given by the fact that the centrifugal chiller control system is one of the most seen in a large variety of applications in HVAC control systems. Since its dynamic model is of high complexity in terms of dimension and encountered nonlinearities, a tight control in closed-loop requires a suitable modelling approach.
\end{abstract}

\section{INTRODUCTION}

' HIS research paper investigates an alternative modelling design methodology for nonlinear systems dynamics, such as an adaptive neuro-fuzzy logic modeling approach, very useful for a large number of control systems applications from different fields, including also multimedia networks and communications. Basically, the new modeling approach is a combination of an artificial neural network (ANN) [1]-[3] and a fuzzy logic (FL) [4]-[6] modeling features. Nowadays considerable advances have been made in applying the ANN systems for problems found intractable or difficult for traditional computation [1]. Some representative preliminary results obtained and related to this field in our research work activity during the years can be found in [3]. The fuzzy logic modelling technique is a powerful tool for the formulation of expert knowledge and the combination of imprecise information from different sources [4]. The FL is in fact a control system modelling technique for a "complicated system without knowledge of its mathematical description", as is also stated in [4]. Fuzzy

This work was not supported by any organization logic modelling technique was applied successfully in our research activity to design an improved hybrid fuzzy sliding mode observer estimator [5]. For simulation purpose and "proof-concept" considerations as a case study is chosen one relevant application from heating ventilation and air conditioning (HVAC) control systems, namely a centrifugal chiller system, that is one of the most widely used in this kind of applications. It is characterized by a great complexity and high nonlinear behavior, as is shown with many details in [7]. As is shown in [7], the centrifugal chillers have become the most widely used devices since they have high capacity, reliability, and require low maintenance. Furthermore, in this recent research paper work is completed a literature review in the field that reveals a significant amount of work done in a classic way on transient and steady state modelling for centrifugal chillers, such is revealed in [8]-[16]. Additionally, we try to complete in the following the literature review with the most recent sophisticated and intelligent approaches related directly to a combined neural networks and fuzzy logic or separately modelling methodologies applied to centrifugal chillers. An interesting integrated modelling methodology is used in [17] to improve the reconstruction of the performance map of axial compressor and fans, where the learning capability of ANN is integrated to the knowledge aspect of fuzzy inference system (FIS) to offer enhanced prediction capabilities rather than using a single methodology independently.

In [18] an application of combined neuro-fuzzy modelling techniques to develop a fault detection, diagnosis and isolation (FDDI) strategy for centrifugal chillers is presented.

A new modelling approach of steady state vapourcompression liquid chillers is presented in [19] that uses a generalized radial basis function (GRBF) ANN to predict chiller performance. As is mentioned also in [7] that centrifugal chillers are the most energy-consuming devices in HVAC applications, especially if they do not operate optimally, i.e. they cannot produce the required cooling load 
capacity, an improvement of their coefficient performance (COP) and to reduce the power consumption will be required. This objective is achieved in [20] by using for model prediction an ANFIS based Fuzzy Clustering Subtractive (FCS) and for classification and optimization an Accelerated Particle Swarm Optimization (APSO) algorithm.

The proposed technique "reduces the total power consumption by $33.2 \%$ and meets the cooling demand requirements", as is stated also in [20]. Also, "it improves the cooling performance based on COP, thus resulting in a $15.95 \%$ increase in efficiency compared to the existing cooling system". The studied ANFIS-based FCS outperforms the ANFIS-based fuzzy C-means clustering in terms of the regression. Then, the algorithm-based classifier APSO has better results compared to the conventional particle swarm optimization (PSO).

Thus, it is important to explore new modelling methodologies for HVAC centrifugal chillers dynamic systems. With this as motivation, the remainder of the paper is structured as follows. In Section 2 the ARX model of the centrifugal chiller based on the measured input-output measurements data set obtained by extensive simulations in open-loop is presented. In Section 3 the simulation results of both closed-loop control subsystems, evaporator and condenser based on ARX models built in Section 2 are shown. In Section 4 the neuro - fuzzy model of centrifugal chiller based on the same open-loop input-output measurements data set used in Section 2 is presented. In Section 5 performance analysis of both models are compared in terms of modeling accuracy. Finally, the Section 6 concludes the relevant contributions of this research paper.

\section{THE ARX MODELS OF CENTRIFUGAL CHILLER}

\section{A. The Simulink Model of the Open Loop Centrifugal Chiller}

Basically, a centralized centrifugal chiller control system can be considered as an interconnection of two main closedloops control subsystems, the first one is a chilled water temperature control loop inside an evaporator, and the second one is a refrigerant liquid level control loop inside a condenser [7]. Since its dynamic model is of high complexity in terms of dimension and encountered nonlinearities, a tight control of the both closed-loops requires a suitable modelling approach. The centrifugal chiller efficiency can be improved by implementing advanced model-based controller design strategies. Consequently, the development of highfidelity centrifugal chiller dynamic model has become a priority task of our research. For interested readers a complete dynamic model of centrifugal chiller under our consideration is given in Annex 1 of our research work [7], pp. 299-305.

In first step of the control design, based on the mentioned modelling development, a MATLAB SIMULINK model for a centrifugal chiller centralized system in open-loop is built, as is shown in Fig. 1 and Fig. 2, similar to those introduced in [7], p. 286.

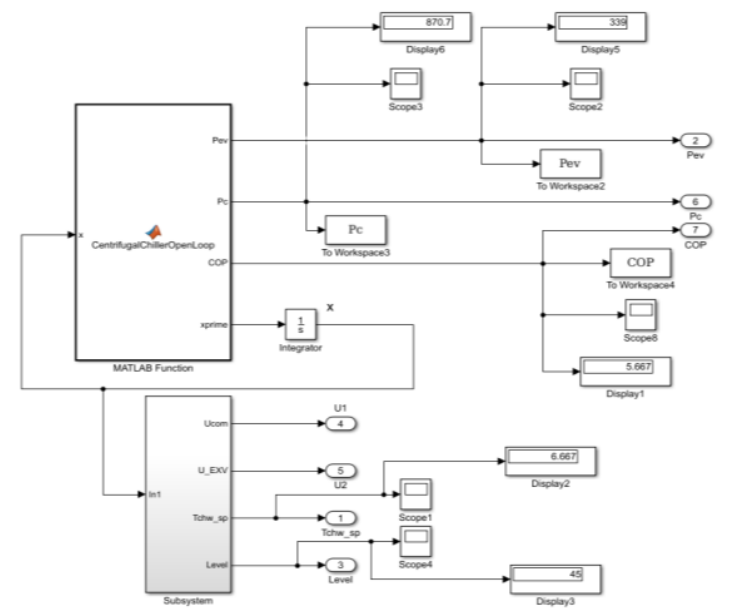

Fig.1 SIMULINK model of centralized centrifugal chiller in open-loop (see also (7), p. 286)

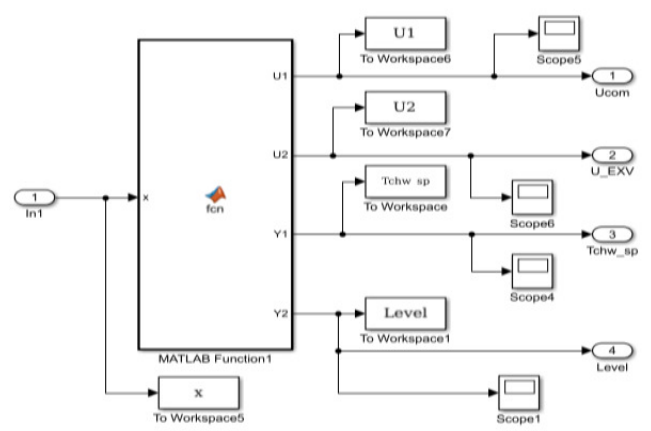

Fig.2 SIMULINK model of the bottom Subsystem block (see also (7), p. 286)

The SIMULINK centrifugal chiller open-loop model is an useful architecture structure support for ARX models, and also to build several closed-loop control strategies as the one based on two single input - single output (SISO) ARX models described in the next section for the overall system, in fact a distributed system, since in "real-life" there exist some interferences between the both loops [7]. Through extensive open-loop simulations is generated the most appropriate input-output data set required to build the linear SISO polynomial ARX models for both open-loops of the centrifugal chiller, as is shown in Fig. 3 for chilled water temperature and Fig.4 for refrigerant liquid level respectively. Consequently, a good open-loop model capable to capture entire dynamics of the overall chiller system under various operating conditions is essential to give more flexibility for closed-loop control design strategies.

\section{B. The SISO ARX Model of Temperature and Liquid Refrigerant Level Open-Loops}

Basically, the arx MATLAB function [7]-[8] estimates the parameters of two polynomials discrete-time models known as autoregressive with an exogenous input (ARX) and a more simple polynomial autoregressive without exogenous input (AR) that estimates the parameters of the scalar time 
series, by means of the well-known least squares method the most used in control systems identification and parameters estimation, such as those well documented in [7]-[8]. Theoretically, the arx MATLAB function uses a predictionerror method and specified polynomial orders [7]-[8]. Also, a pure transport delay of the signal flow in the feedback path from the measurement sensors to the controllers is specified in each ARX structure. The ARX model is "inherently linear and the most significant advantage is that we can perform model structure and parameter identification rapidly", as is stated in [9]. To get the ARX model the data set is divided in two segments, first segment consisting of 1000 samples required for the prediction phase, and the second one consisting of the remaining 2600 samples required for validation phase respectively. The information obtained on the discrete-time chilled water temperature ARX model in MATLAB command window is:

$A(z) y(t)=B(z) u(t)+e(t)$

where the coefficients of the polynomials $A(z), B(z)$ are estimated by the well-known least square errors (LSE) procedure, given in the next two equations:

$$
\begin{aligned}
& A(z)=1-1.096 z^{-1}-0.7723 z^{-2}+0.8689 z^{-3} \\
& B(z)=7.98 z^{-2}-8.074 z^{-3}-8.775 z^{-4}+8.31 z^{-5}+\ldots \\
& 2.009 z^{-6}-1.44 z^{-7}+0.002313 z^{-8}-0.009972 z^{-9}+\ldots \\
& -0.002079 z^{-10}+0.001031 z^{-11}
\end{aligned}
$$

Sample time: 1 second

Parameterization:

Polynomial orders:

$n_{a}=3(A(z)$ degree $), n_{b}=10(B(z)$ degree $)$,

$n_{k}=2$ (delay)

Number of free coefficients: 13

Status:

Estimated using ARX on time domain data.

Fit to estimation data: $100 \%$ (prediction focus)

FPE: 2.821e-11, MSE: 2.694e-11

The argument variable $z^{-1}$ is the equivalent of the timediscrete delay operator $q^{-1}$, as is shown in [7]-[8], i.e.

$z^{-1} y(t)=q^{-1} y(t)=y(t-1)$, and $e(t)$ is an additive white noise.

The simulation results obtained in both prediction phase (in the top graph side) and validation phase respectively (in the bottom graph side) are shown in Fig.5. These results reveal a good performance in the both phases for chilled water temperature. Similarly, for refrigerant liquid level SISO ARX open-loop the following information provided in MATLAB command window is very useful for controller design:

$$
A(z) y(t)=B(z) u(t)+e(t)
$$

$$
\begin{aligned}
A(z)= & 1-2.02 z^{-1}+0.5349 z^{-2}+0.8555 z^{-3}+\ldots \\
- & 0.2345 z^{-4}+0.08802 z^{-5}-0.4596 z^{-6}+\ldots \\
& 0.1761 z^{-7}+0.131 z^{-8}-0.07358 z^{-9}+\ldots \\
& 0.001818 z^{-10} \\
B(z)= & -15.85 z^{-1}+19.63 z^{-2}+4.138 z^{-3}-3.039 z^{-4}+\ldots \\
- & 8.471 z^{-5}-0.677 z^{-6}+5.626 z^{-7}-0.2662 z^{-8}+\ldots \\
- & 1.1 z^{-9}
\end{aligned}
$$

Sample time: 1 seconds

Parameterization:

Polynomial orders:

$n_{a}=10(A(z)$ degree $), n_{b}=9(B(z)$ degree $)$,

$n_{k}=1$ (delay)

Number of free coefficients: 19

Status:

Estimated using ARX on time domain data.

Fit to estimation data: $99.98 \%$ (prediction focus)

FPE: 6.911e-08, MSE: 6.502e-08

The simulation results obtained in both prediction phase (in the top graph side) and validation phase respectively (in the bottom graph side) are shown in Fig.6. These results reveal a poor performance in the both phases for refrigerant liquid level, caused by the presence of the oscillations around the level set point of $45 \%$ of the liquid in Condenser.
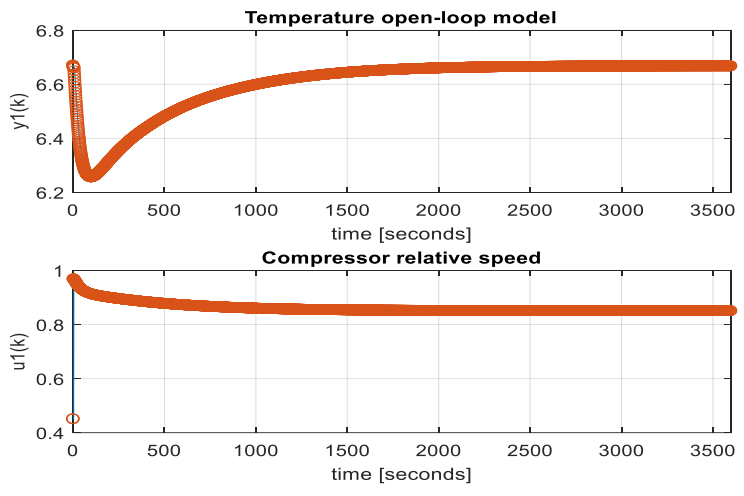

Fig.3 Temperature open-loop model

Concluding, the performance in Fig. 7 and Fig. 8 appears to be very good for chilled water temperature, and worst for refrigerant liquid level. However, if a better performance level is desired, we might want to switch to a nonlinear model. In particular, we are going to use a neuro-fuzzy modeling approach ANFIS, to see if we can push the performance level of an off-line trained ANN with a fuzzy inference system (FIS), as can be seen in Section 4. 

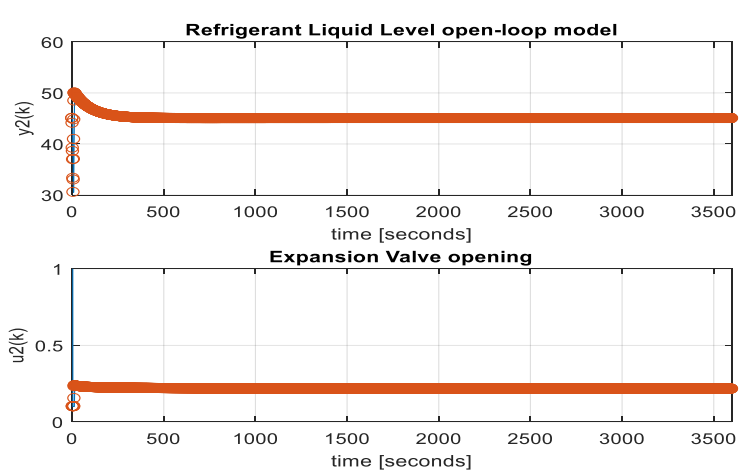

Fig.4 Refrigerant liquid level open-loop model

(a) Training Data (Solid Line) and ARX Prediction (Dots)

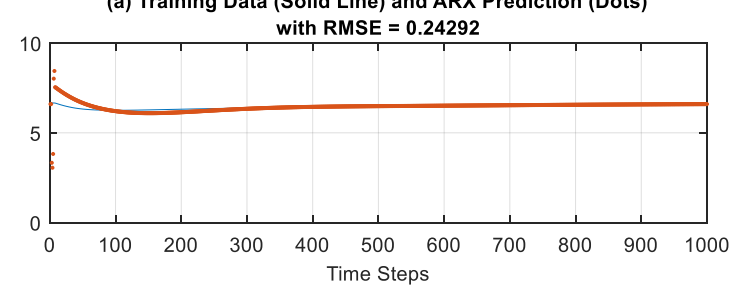

(b) Checking Data (Solid Line) and ARX Prediction (Dots)

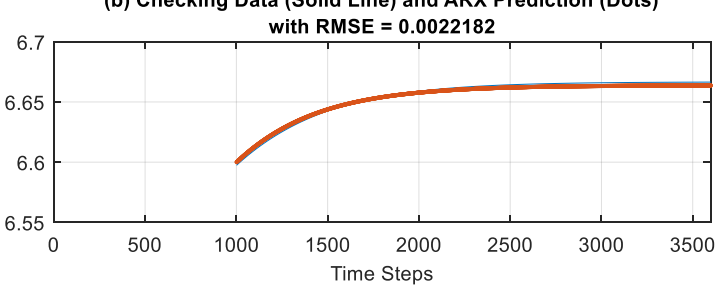

Fig.5 Temperature SISO ARX open-loop model

(a) Training Data (Solid Line) and ARX Prediction (Dots)

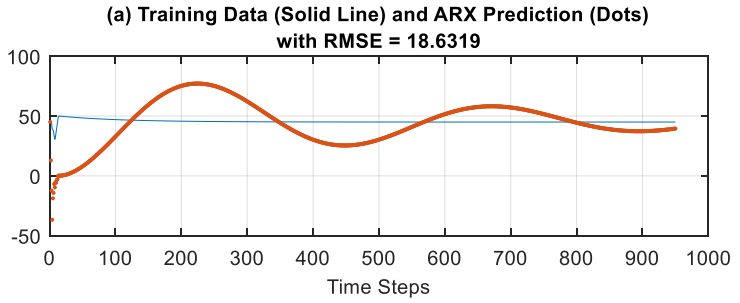

(b) Checking Data (Solid Line) and ARX Prediction (Dots) with RMSE $=1.5372$

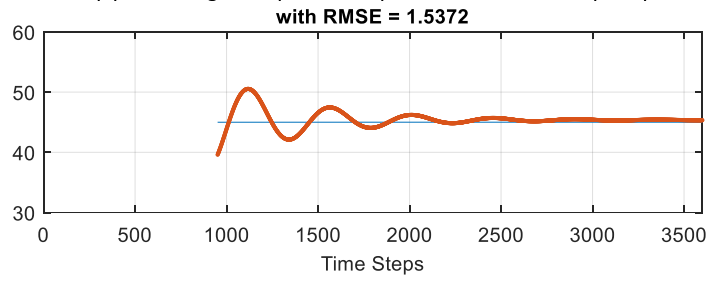

Fig.6 Refrigerant liquid level SISO ARX open-loop model

\section{Centrifugal ChILler SiSO ARX Models Closed- LOOP SIMULATION RESULTS}

In this section we show the great potential of both ARX models for control design in feedback closed-loop. Precisely, we show the results of the standard proportional integral derivative (PID) control used in each closed-loop to control the chilled water temperature and the refrigerant liquid level. The main idea is that even if the ARX models performance is not the best one, but it is satisfactory, two well-tuned PID controllers, i.e. their parameters $k_{P}, k_{I}$, and $k_{D}$ have optimal values, as is shown also in [7], compensate the modeling mismatches, by performing very well in closed-loop. The Simulink model of the both closed-loops is shown in Fig. 7, and the simulation results that reveal a very good performance in terms of robustness to the changes in set points and convergence with a very fast transient are shown in Fig. 8, for chilled water temperature, and Fig.9 for refrigerant liquid level.

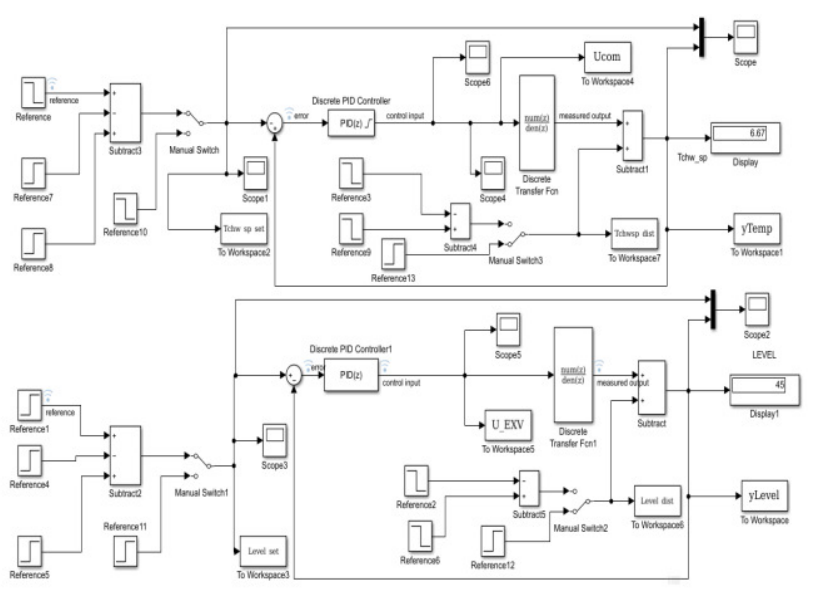

Fig.7 SIMULINK SISO ARX model of centrifugal chiller in closed-loop

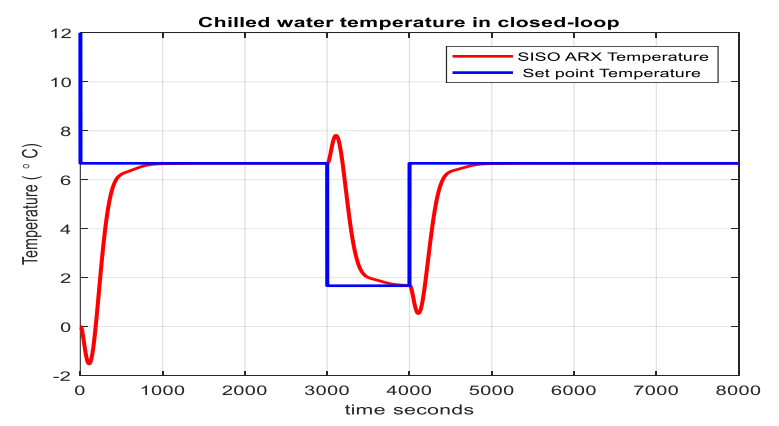

Fig.8 Chilled water Temperature SISO ARX closed-loop model

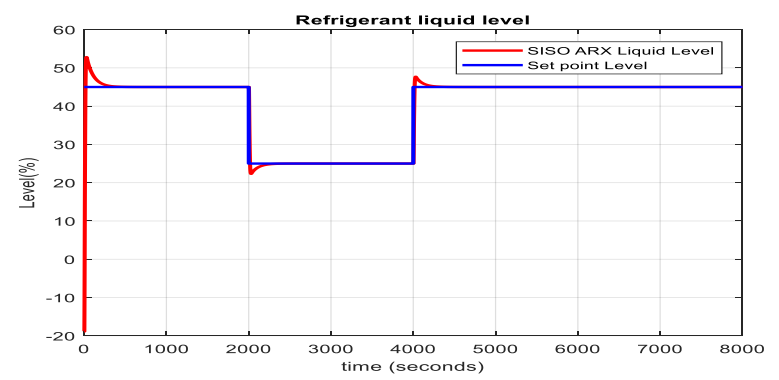

Fig.9 Refrigerant liquid level SISO ARX closed-loop model

\section{THE ANFIS MODEL OF CENTRIFUGAL CHILLER}

Basically, the architecture of an adaptive neuro-fuzzy inference system (ANFIS) attached to the centrifugal chiller nonlinear model is a combination of an ANN that drives a fuzzy inference system (FIS). The ANN is trained online or offline based on an input-output measurements data set collected as a result of extensive open-loop simulations to find the most appropriate nonlinear relationship between the inputs and the outputs of the both open-loops of centrifugal chiller control system. 
Essentially, the proposed ANN structure has the following three main features:

- Ability to find the solution of such problems for which algorithmic method is expensive or does not exist

- Ability to learn by experience, thus is not need to program them at much extent

- High accuracy and a considerable fast processing speed than traditional systems.

The proposed ANN computation is performed by "of dense computing mesh nodes and connections" [1], as is shown in Fig. 10 [2]. It is a driven data based, operating "collectively and simultaneously" [1], [3] to learn (e.g. in supervised or un-supervised learning mode) how to match the input and output training (offline or online) measured data set (prediction phase) of the centrifugal chiller control system under consideration, thus to establish an accurate relationship (i.e. model), validated on the supplemental segment data set (validation phase). The basic processing elements of the proposed ANN structure are called artificial neurons, or simply nodes, that typically operate in parallel and are configured in regular architectures, similar to those shown in Fig. 10.

Within the ANN architecture the neurons perform similar to summing and nonlinear mapping junctions.

Basically, the ANN architecture structure contain input, hidden and output layers, as well as several feedback connections within the layer and toward adjacent layers.

Each connection strength is expressed by a numerical value called weight, which can be modified during the online or offline training in a prediction phase of computational process.

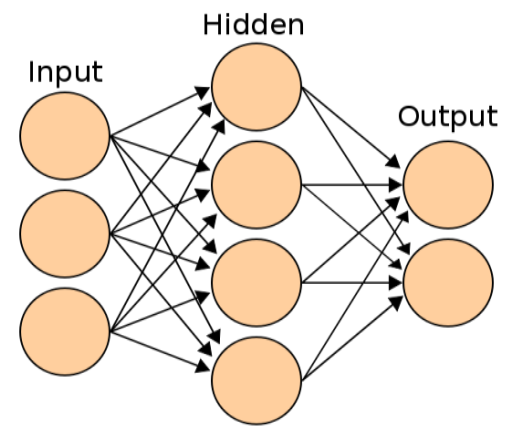

Fig.10 A simple ANN architecture (reproduced from [2])

Fuzzy logic modelling technique is a "computational paradigm that is based on how humans think", as is stated in [6]. According also to [6] in fuzzy logic, any statement assumes a probabilistic value between 0 and 1, representing the membership degree of an element that belongs to a given set.

The first step in ANFIS modeling system identification is the input selection to determine which variables should be the input arguments to the ANFIS model. For simplicity, as is suggested in [9] we assume that there are 10 input candidates for the ANN [3]:

$$
\begin{aligned}
& (y(k-1), y(k-2), y(k-3), y(k-4)), \\
& (u(k-1), u(k-2), u(k-3), u(k-4), u(k-5), u(k-6))
\end{aligned}
$$

and the output to be predicted is $y(k)$. A heuristic approach to input selection is called sequential forward search, in which each input is selected sequentially to optimize the total squared error (RMSE). This can be done by using the MATLAB function seqsech; the results are shown in Fig. 11 for chilled water temperature, and Fig.12 for refrigerant liquid level respectively.

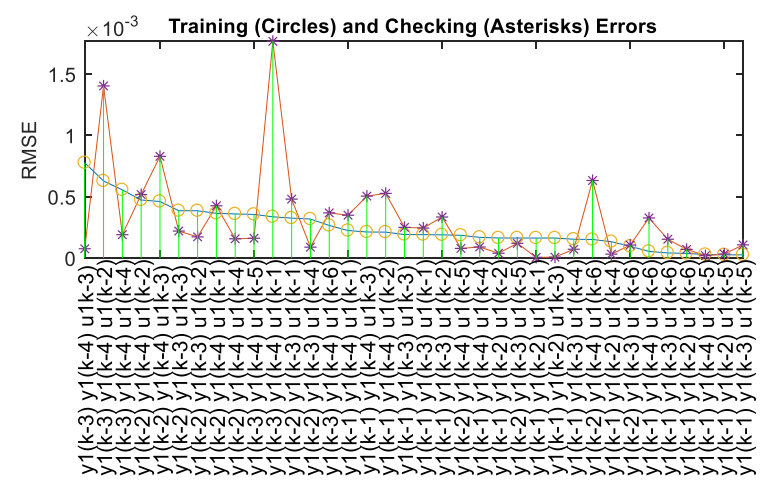

Fig.11 Temperature SISO ANFIS open-loop model training phase Legend: ANFIS info

Number of nodes: 34

Number of linear parameters: 32

Number of nonlinear parameters: 18

Total number of parameters: 50

Number of training data pairs: 1000

Number of checking data pairs: 2601

Number of fuzzy rules: 8

Minimal training RMSE $=0.000025$

Minimal checking RMSE $=0.000120906$

$\left[\begin{array}{lll}n_{a} & n_{b} & n_{k}\end{array}\right]=10 \quad 9 \quad 1$

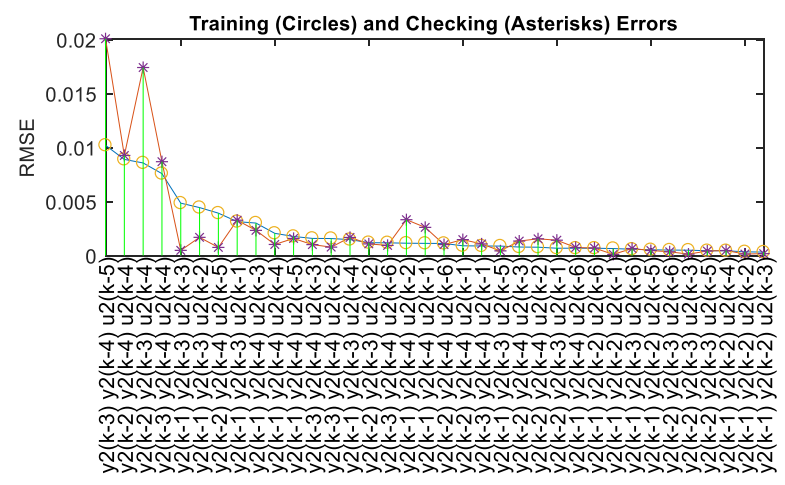

Fig.12 Refrigerant liquid level SISO ANFIS open-loop model training

Legend: ANFIS info phase

Number of nodes: 34

Number of linear parameters: 32

Number of nonlinear parameters: 18

Total number of parameters: 50

Number of training data pairs: 950

Number of checking data pairs: 2651

Number of fuzzy rules: 8

Minimal training RMSE $=0.000320$

Minimal checking RMSE $=0.000274694$ 


$$
\left[\begin{array}{lll}
n_{a} & n_{b} & n_{k}
\end{array}\right]=10 \quad 9 \quad 1
$$

The inputs are selected with a training RMSE of 0.000320 , and checking RMSE of 0.000120906 , for chilled water temperature, and a training RMSE of 0.000274694 , for refrigerant liquid level respectively.

The simulation results for the estimated nonlinear centrifugal chiller ANFIS models in open-loop are shown in Fig.13, for chilled water temperature, and Fig.14 for refrigerant liquid level.
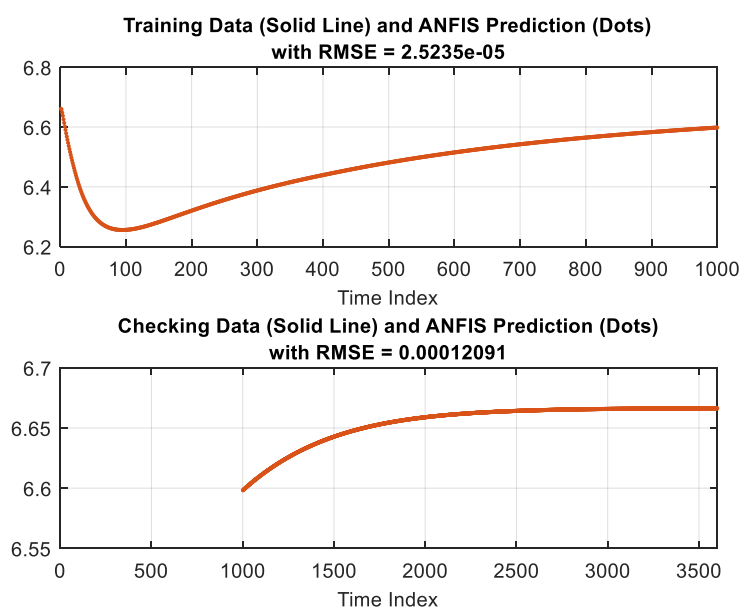

Fig.13 Temperature SISO ANFIS open-loop model

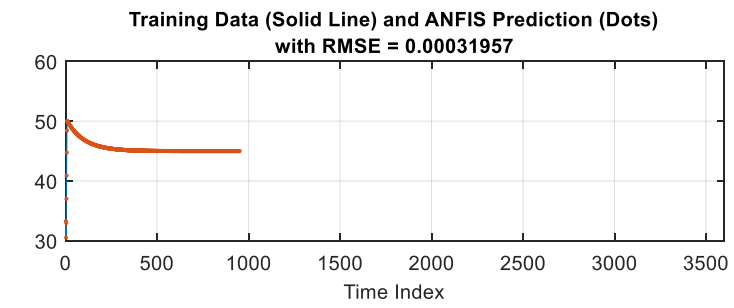

Checking Data (Solid Line) and ANFIS Prediction (Dots)

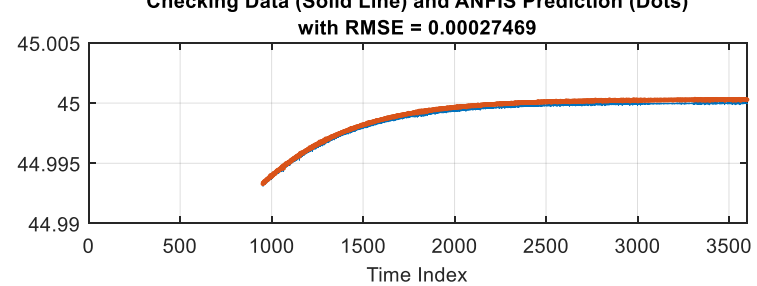

Fig.14 Refrigerant liquid level SISO ANFIS open-loop model

As mentioned in Section 2 for ARX models we follow the same procedure to split the original data set obtained by extensive open-loop simulations in two data subsets, the first one for training phase (the top side of the graphs), and the second one for prediction (estimation) phase (the bottom side of the graphs) respectively. These modeling aspects can be seen also in Fig.13 and Fig.14. The simulation results reveal a great accuracy modeling performance for the nonlinear ANFIS models compared to the performance of linear ARX models, and thus they have also a great potential for control design.
To simplify the ANFIS modeling design we suggest the use of the MATLAB function anfis provided by MATLAB Fuzzy Logic Toolbox, that is a hybrid learning algorithm to identify the membership function parameters of single output, Sugeno type FIS. A combination of LSE and backpropagation gradient descent methods [3] are used for training FIS membership function parameters [5] to model a given set of input-output data set, as is mentioned in MATLAB Fuzzy Logic Toolbox [8].

\section{V.CONCLUSION}

This research paper is an interesting MATLAB application of a modeling design approach that uses the most accurate nonlinear adaptive neuro-fuzzy ANFIS models, as an alternative to linear polynomial ARX models. If we want to choose between ARX and ANFIS models we need to think in terms of fast implementation or precision.

For fast implementation we can choose the ARX models since these are inherently linear and the most significant advantage is that we can perform model structure and parameter identification rapidly. If a high modeling accuracy performance is desired, we are going to use a neuro-fuzzy modeling approach ANFIS, to push the performance level with a fuzzy inference system. In the future work we will extend the investigations area to apply the nonlinear ANFIS models for many other similar applications in different fields, and to explore their great potential in closed-loop control systems, and also in sliding mode control [3], [10],[23].

\section{REFERENCES}

[1] J.M. Zurada, Introduction to artificial neural networks, $1^{\text {st }}$ ed. Ed. St. Paul, USA, MN: West Publishing company, 1992, ISBN 0-3 14$93391-3$.

[2] [Internet]. Wikipedia. Available online on the website: https://en.wikipedia.org/wiki/Artificial_neural_network, accessed at May $2^{\text {nd }} 2018$.

[3] M. Zaheeruddin, N. Tudoroiu, "Neuro - PID tracking control of a discharge air temperature system", Elsevier, Energy Conversion and Management, vol. 45, pp.2405-2415, 2004, DOI:10.1016/j.enconman.2003.11.016.

[4] B. Bouchon-Meunier, M. Detyniecki, M-J. Lesot, C. Marsala, M. Rifqi, "Real-World Fuzzy Logic Applications in Data Mining and Information Retrieval". Available on website at: https://pdfs.semanticscholar.org/b86c/2c39d3457bd439b2e280eff813 4768fcbe90.pdf, accessed at May $2^{\text {nd }} 2018$.

[5] S.M. Radu, E-R Tudoroiu, W. Kecs, N. Ilias, N. Tudoroiu, "Real Time Implementation of an Improved Hybrid Fuzzy Sliding Mode Observer Estimator", Advances in Science, Technology and Engineering Systems Journal, vol. 2, no. 1, January 2017, pp.214226, ISSN: 2415-6698, DOI: 10.25046/aj020126, www.astesj.com.

[6] Fuzzy Logic Tutorial, available online on the web site: http://www.massey.ac.nz/ nhreyes/MASSEY/159741/Lectures/Lec20 12-3159741-FuzzyLogic-v.2.pdf.

[7] N. Tudoroiu, M. Zaheeruddin, S. Li, E-R. Tudoroiu, "Design and Implementation of Closed-loop PI Control Strategies in Real-time MATLAB Simulation Environment for Nonlinear and Linear ARMAX Models of HVAC Centrifugal Chiller Control Systems", Advances in Science, Technology and Engineering Systems Journal, vol. 3, no. 2, April 2018, pp.283-308, ISSN: 2415-6698, DOI: 10.25046/aj030233,www.astesj.com. 
[8] S. Bendapudi, J. E. Braun, et al., "Dynamic Model of a Centrifugal Chiller System - Model Development, Numerical Study, and Validation", ASHRAE Trans., vol. 111, pp.132-148, 2005.

[9] A. Beyene, H. Guven, et al., (1994)," Conventional Chiller Performances Simulation and Field Data", International Journal of Energy Research, vol.18, pp.391-399, 1994.

[10] J. E. Braun, J. W. Mitchell, et al., "Models for Variable-Speed Centrifugal Chillers", ASHRAE Trans., New York, NY, USA, 1987.

[11] M. W. Browne, P. K. Bansal, "Steady-State Model of Centrifugal Liquid Chillers", International Journal of Refrigeration, vol. 21, no.5, pp. 343-358, 1998.

[12] J.M. Gordon, K. C. Ng, H.T. Chua, "Centrifugal chillers: thermodynamic modeling and a diagnostic case study", International Journal of Refrigeration, vol.18, no. 4, pp.253-257, 1995.

[13] Li Pengfei, Li Yaoyu, J. E. Seem, "Modelica Based Dynamic Modeling of Water-Cooled Centrifugal Chillers", International Refrigeration and Air Conditioning Conference, Purdue University, pp.1-8, 2010. http://docs.lib.purdue.edu/iracc/1091.

[14] P. Popovic, H. N. Shapiro, "Modeling Study of a Centrifugal Compressor", ASHRAE Trans., Toronto, 1998.

[15] M. C. Svensson, "Non-Steady-State Modeling of a Water-to-Water Heat Pump Unit", in Proceedings of $20^{\text {th }}$ International Congress of Refrigeration, Sydney, 1999.

[16] H. Wang, S. Wang, "A Mechanistic Model of a Centrifugal Chiller to Study HVAC Dynamics”, in Building Services Engineering Research and Technology, vol. 21, no.2, pp. 73-83, 2000.
[17] M. Gholamrezaei and K. Ghorbanian, "Application of integrated fuzzy logic and neural networks to the performance prediction of axial compressors", J Power and Energy, vol. 229(8), pp. 928-947, 2015, DOI: $10.1177 / 0957650915596877$.

[18] Q. Zhou, S. Wang, F. Xiao, “A Novel Strategy for the Fault Detection and Diagnosis of Centrifugal Chiller Systems", HVAC\&R RESEARCH Journal, vol. 15(1), pp.57-75, 2009.

[19] D. J. Swider, M.W. Browne, P. Bansal, V. Kecman, "Modelling of vapour-compression liquid chillers with neural networks", Applied Thermal Engineering Journal, vol. 21(3), pp. 311-329, 2001, DOI: 10.1016/S1359-4311(00)00036-3.

[20] Elnazeer Ali Hamid Abdalla, Perumal Nallagownden, Nursyarizal Bin Mohd Nor, Mohd Fakhizan Romlie, Sabo Miya Hassan, "An Application of a Novel Technique for Assessing the Operating Performance of Existing Cooling Systems on a University Campus", Energies Journal, vol.11(4), 719, 2018; DOI:10.3390/en11040719.

[21] [Internet]. Available online on website: https://www.mathworks.com/help/ident/ref/armax.html, MATLAB R2017b Documentation. Accessed at February 3rd, 2018.

[22] [Internet]."ANFIS Model Identification", available online on website: https://www.mathworks.com/help/fuzzy/examples/nonlinear-systemidentification.html\#d119e1365. Accessed at March 25 th 2018.

[23] R-E Tudoroiu, W. Kecs, M. Dobritoiu, N. Ilias, S-V Casavela, N.Tudoroiu," Real-Time Implementation of DC Servomotor Actuator with Unknown Uncertainty using a Sliding Mode Observer", ACSIS, vol.8, pp.841-848,DOI: 10.15439/2016F95, Poland, 2016. 